\title{
Advanced Control Strategies For The Modulation of Solar Radiation In Buildings: MPC- enhanced Rule-based Control
}

\author{
Marco Savino Piscitelli ${ }^{1}$, Silvio Brandi ${ }^{1}$, Giovanni Gennaro ${ }^{1}$, Alfonso Capozzoli ${ }^{1}$, Fabio Favoino ${ }^{1}$, \\ Valentina Serra ${ }^{1}$
}

\author{
${ }^{1}$ Technology Energy Building Environment research group, Department of Energy, Politecnico di \\ Torino, Italy
}

\begin{abstract}
The present work is aimed at exploring the potentials of extracting control rules of a smart glazing from an optimal control strategy obtained by means of an ideal model predictive controller (MPC). To this sake an ideal deterministic MPC (Model Predictive Control with ideal prediction of disturbances), minimising total energy use, is devised for the control of a smart glazing for a Southoriented enclosed office space in London. Secondly, a data mining-based method is adopted to extract sets of rules from the simulated MPC-controlled data set. Finally, these rules are compared with the ideal MPC performance, and with reference threshold-based control rules.
\end{abstract}

\section{Introduction}

Appropriate integration of smart glazing technologies in building can significantly contribute towards the achievement of decarbonisation targets while maintaining adequate levels of thermal and daylight comfort in the built environment (Aelenei et al. 2019). The control of adaptive façades, including switchable glazing, can be a self-triggered mechanism (passive/smart control) or it can be triggered by an external stimulus (active/intelligent). Active technologies include electrochromic (EC), suspended particle (SPD), and liquid crystal (LCD) devices, in which the modulation of an electrical (for electron injection/removal, or variation of magnetic field) across a transparent conductor induces a variation of the optical properties of the functional layer. During building operations, an adaptive glazing can be controlled to meet multiple (and sometimes conflicting) performance requirements. Therefore, smart glazing performance is highly dependent on the control strategies adopted during building operations (Favoino et al., 2016). For these reasons the design of optimal control strategies for smart glazing technologies is still a significant challenge, strongly influencing their building integration (Lee et al. 2012).

Many researchers investigated the influence of control strategies on the performance of adaptive glazing technologies. Assimakopoulos et al. (2004), Assimakopoulos et al. (2007), Guglielmetti and Bisegna (2003), Jonsson and Ross (2010), Lee and Tavil (2007), show how the switching settings of EC windows can dramatically change indoor comfort levels and energy use of buildings. In general, the tested control strategies are RBC (Rule Based Control).

$\mathrm{RBC}$ is by far the most adopted control option in the market (Oldewurtel et al. 2012). These are based on simple "if-then" rules adopting single or multiple fixed pre-determined set-points, relative to limited measurements of environmental boundary conditions. The most used parameters in active smart glazing controls are (Favoino et al. 2016): a) solar geometry; b) outdoor horizontal or vertical illuminances; c) outdoor horizontal or vertical solar radiation d) indoor daylight conditions; e) presence of occupants; f) temperature difference between outdoor and indoor; g) presence of heating / cooling loads; h) seasonal building services set-points. Only a small number of cases have explored more advanced controls strategies such as: combination of the above mentioned rules/ parameters (Lee et al. 2012); PI and PID controls based on indoor illuminances; fuzzy logic controls based on occupant preferences and environmental conditions (indoor illuminances and temperature, external vertical solar radiation) (Assimakopoulos et al. 2007).

Very few studies compared the performance of rule-based control (RBC) strategies for smart glazings with strategies which are based on an online optimisation of a certain performance indicator (Favoino et al. 2016, Dussault et al. 2012). Moreover, these research studies highlighted the importance of predicting of the effect of the entering solar radiation in the optimisation of smart glazing controls. In particular Favoino et al. (2016) showed as a Receding Horizon Control (RHC), including the prediction of the influence of entering solar radiation on the future energy balance of a building, can increase significantly the energy saving achievable by means of a smart glazing, as compared to an optimised rule based control based on current and past conditions The highest energy savings are realised when the modulation of solar radiation is able to balance the heating or cooling energy use of the building, while in more extreme cooling or heating conditions, the performance of an RHC control is very similar to an RBC.

Nevertheless, real-world implementation of online optimised predictive control strategies (also referred to as Model Predictive Control, MPC) would involve a higher cost compared to RBC ones, as well as a bigger challenge in ensuring the predicted performance. In fact, this would require a calibrated building model, forecasts of weather and endogenous loads, and a larger number of sensors in order to update the model according to the acquired 
measurements (Wang and Ma, 2008). Jin et al. (2017) observed that MPC applied to control of an active building envelope system, often requires less control actions than an RBC one which, from a first qualitative analysis, may be related to external boundary conditions, such as outdoor temperature and incident solar radiation, internal boundary conditions, such as occupation, endogenous loads and air temperature set points, and/or to building states, such as indoor air temperature and wall internal surface temperature, depending on the season. Therefore, it may be possible to reduce the complexity of smart glazing MPC controls by extracting simpler rules that can be adopted in operation to mimic optimal control, with a relatively low decrease of operational performance.

The aim of an MPC-enhanced RBC would be reduce the cost of implementing optimised control strategies, reducing the need for a calibrated building model and online optimisation during building operations, and to reducing the number of sensors needed. The main advantage of this procedure consists in developing and updating detailed energy simulations and the optimal MPC control logic during off-line analysis. In this way the control system does not require a high computational power or a set of specific technologies to communicate MPC decision to a Building Automation System that is often not practical in all facilities.

The effectiveness of an MPC rule extraction technique has already been proven by simulation (May-Ostendorp et al. 2011) and with experiments (May-Ostendorp et al. 2013) for thermally active buildings.

In light of the above the present paper aims to propose and test an innovative data mining approach to extract effective control rules, adapting to the variation of boundary conditions, from a simulated MPC for the optimal control of a smart glazing.

Section 2 presents the methodological framework which is proposed, and the data mining techniques adopted. Following a description of the simulated MPC case study, which is adopted to develop and test the present methodological framework. Finally, the performance of the present methodology is presented by comparing the performance of the MPC-enhanced RBC compared to the traditional MPC.

\section{Methodology}

In this section, the methodological framework is presented with the aim of discussing each stage of the process. The methodology is based on the application of classification and regression trees for the extraction of an advanced set of control rules from an MPC control signal. The multistep process has been tested for the control of a smart glazing for a South-oriented enclosed office space in London. The choice of the climate was motivated by the maximisation of the effect of MPC in modulating entering solar radiation, which is higher in heating dominated climates (Favoino et al. 2016). The general framework unfolds over different stages, as shown in Figure 1.
The first stage is aimed at simulating an optimal control strategy obtained by means of an ideal model predictive control (MPC) according to Favoino et al. (2016). The simulated predictive controller minimises the total energy use of the room over the prediction horizon (i.e., cooling, heating, lighting), selecting hour by hour the optimal discrete state of the smart glazing.

Given that the simulated MPC leverages on perfect predictions of disturbances, the obtained energy performance can be considered as the best reference solution achievable.

The outcome of the first stage is then a one-year dataset of optimal hourly control signal of the smart glazing together with other influencing variables concerning both indoor and outdoor conditions. By the way, given that the smart glazing modulates the amount of solar radiation entering the indoor environment, the control signals during night hours (i.e., from 09:00 p.m to 06:00 a.m.) were excluded from the training/testing dataset.

The second stage of the analysis is aimed at extracting from the MPC control logic, a set of decision rules capable to: (i) reduce the complexity and the computational cost in implementing the glazing controller, (ii) achieve an energy performance close to the reference optimal solution, (iii) increase the control logic interpretability. To this purpose a decision tree algorithm (i.e., CART) has been employed. In energy and buildings applications classification and regression trees have been used widely for energy fault detection and diagnosis (Yan et al. 2016), estimation of building energy usage (Capozzoli et al. 2015) as well as energy benchmarking (Capozzoli et al. 2016).

In this case study the decision tree is used to predict the hourly optimal discrete state of the smart glazing (during the morning hours) provided by the MPC controller, exploiting few variables related to indoor and outdoor conditions as predictive attributes. In this study, two different trees have been trained and tested assuming different pools of input variables. The first tree is fed only using backward-lagged variables while in the second, also forward-lagged ones are considered (i.e., hourly average outdoor temperature and solar radiation of the next hours).

The optimal size of the classification and regression trees has been assessed through a cost-complexity process, searching for a trade-off between the misclassification error of the predicted discrete states of the glazing and the number of decision rules extracted. In this stage the performance of both decision trees is assessed by an open loop test evaluating their capability in reproducing the MPC control sequence. The third stage of the analysis is aimed at comparing the extracted control logics versus the MPC and other rule-based controllers through a closed loop test embedding them in the building energy model.

This case represents the closest approximation of the controller performance on a real automation system. In fact, the misclassification error made by the controller could cause the occurrence of indoor conditions different from those experienced during the training phase generating a divergence from the optimal solution. 
Thanks to this analysis it is possible to assess if the prediction error of the optimal control state of the glazing corresponds to an acceptable reduction of the simulated energy performance. In addition, other post mining analyses were conducted, in order to better describe and characterize the control logic also discussing its limits and strengths in being generalized.

\section{Classification and Regression Tree}

Classification and regression trees are machine-learning algorithms aimed at developing descriptive and/or predictive models from a set of records. Each record is a tuple $(\mathrm{x}, \mathrm{y})$, where $x$ represents the predictive attribute set while $y$ is the target attribute. Depending on the target attribute it is possible to distinguish classification from regression trees. In particular, tree models designed for a categorical target attribute (e.g., the discrete state of the smart glazing) are called classification trees, otherwise if $y$ is a continuous numerical attribute, they are defined as regression trees (Tan et al. 2005). In this work, the CART algorithm has been employed for addressing a predictive modelling task. CART is a specific machine learning technique that is based on the recursive binary splitting of the records into purer subsets called nodes.

In this way, the decision tree can be represented by means of a hierarchical structure that consists of nodes and directed edges (i.e., branches). The final nodes (i.e., leaves) represent the predicted class labels of the target attribute, and the branches represent the conjunctions of the predictive attributes that lead to those classes.

The development of a classification tree, as in all predictive models, unfolds in two main steps: training and testing of the model. k-fold cross-validation has been used in this paper with $\mathrm{k}=10$. In $\mathrm{k}$-fold cross-validation, the dataset is divided into $k$ equal sized subsamples. One of the $\mathrm{k}$ subsets is then used as the validation set and the other k-1 subsets are put together for the training. This process is then repeated $k$ times, using a subsample at a time for the testing. The error estimation is averaged over all $\mathrm{k}$ trials to get total effectiveness of the classification model. In this way, every record is included in a validation set exactly once, and in the training set k-1 times.

For each iteration (the number of iterations is equal to $\mathrm{k}$ ), all the records are initially grouped in one node (i.e., root node) and the algorithm evaluates the best recursive segmentation of the dataset, using the attribute that minimises the average impurity measure (e.g., GINI index, Entropy) of the child nodes after the splitting. In order to avoid model overfitting, early stopping criteria can be set by the analyst (e.g., minimum number of cases in parent and child nodes, maximum tree depth, minimum reduction in node impurity after splitting).

Even though the early stop criteria have been satisfied, the decision tree may continue to be quite complex. For this reason, the cost-complexity pruning process (i.e., 1-SE rule) was performed. This procedure makes it possible to identify the optimal value of the cost-complexity parameter $\alpha$ in order to prune the original tree obtained by the cross-validation process. The procedure selects the simplest subtree (i.e., with the minimum number of final nodes) that can be considered statistically equivalent to the fully grown one in terms of learning rate (Capozzoli et al. 2018).

\section{Model and case study}

The aim of the present study is to compare the performance of controlling a smart glazing by means of an MPC, specifically of an optimised deterministic predictive control (with perfect knowledge of future boundary conditions) and an RBC control, derived from the MPC control by means of datamining techniques (rule extraction).

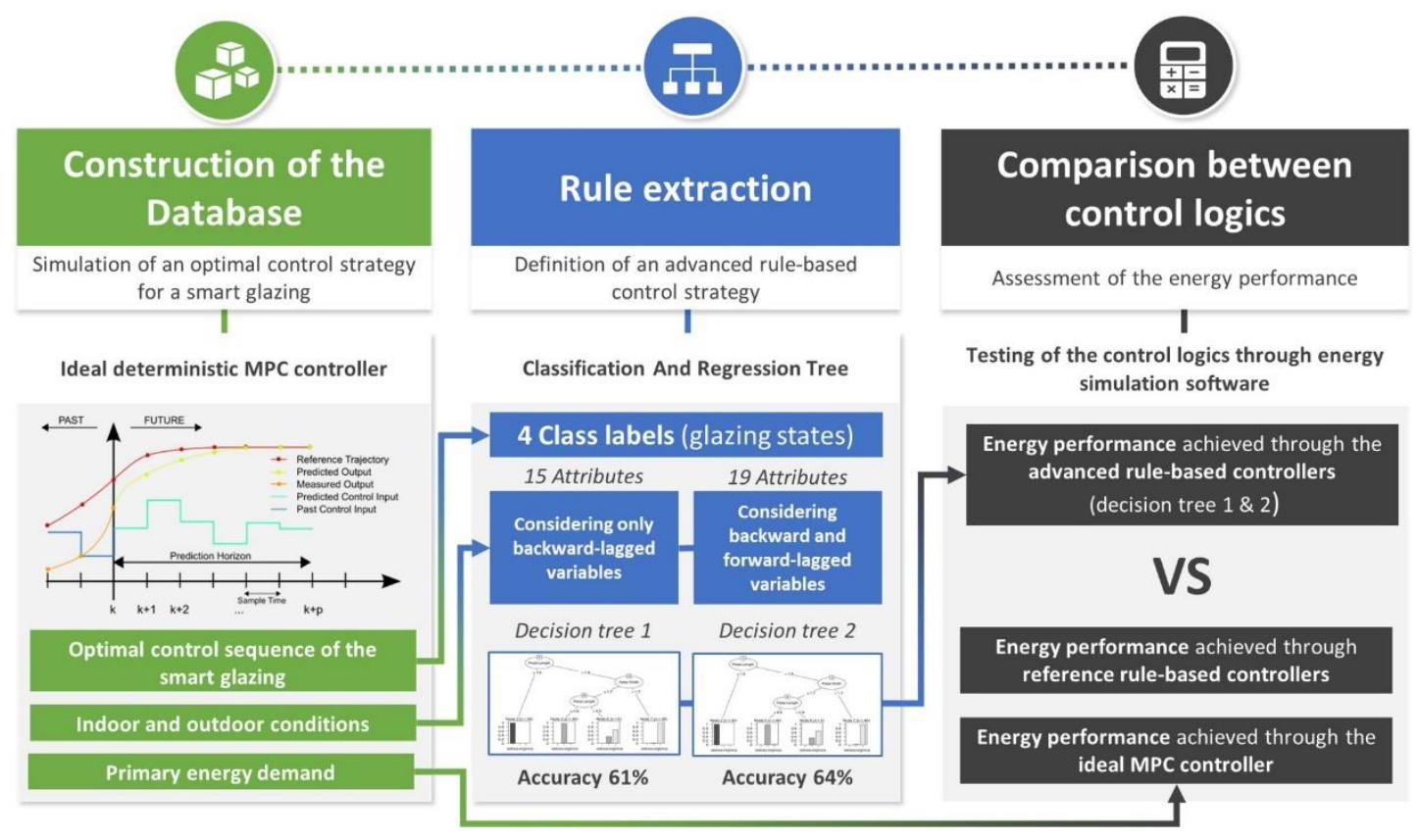

Figure 1: Framework of the analysis. 
A simulated case study is adopted to compare the alternative control methodologies and to test the performance of the presented methodology.

The performance related to energy use only is targeted, although it might be possible to include in the present methodological framework multiple constraints related to improvement of comfort conditions. Therefore, the total specific yearly Site Energy (SE) is used as performance indicator, measuring the total specific amount of energy which is delivered to the building, and used for heating, cooling and lighting purposes (1):

$$
S E=E_{h}+E_{c}+E_{l} \quad\left[k W h / m^{2} y\right]
$$

The data related to the case study, which is briefly presented in this section, are taken from (Favoino et al. 2016).

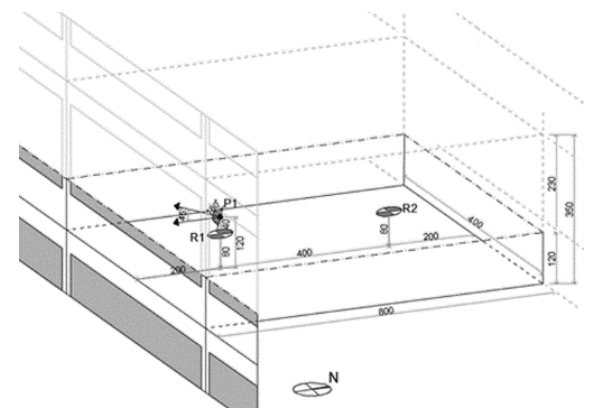

Figure 2: Enclosed office reference room.

The virtual test case building is a reference enclosed office room (4 m wide $\times 8 \mathrm{~m}$ deep $\times 3.5 \mathrm{~m}$ high) in a heating dominated climate (i.e. London), with a Windowto-Wall-Ratio of $60 \%$ on the South-oriented façade (Figure 2). EnergyPlus version 8.3.3 is adopted for the thermal model and to perform the energy simulations (DOE U.S. 2014). The simulation algorithms in EnergyPlus have been chosen to achieve a balance between accuracy and a reasonable computational time of a single simulation run (solar calculations 15 days, conduction transfer function method with a 10-minute time step, adaptive convection algorithm, initialization period 25 days). The characteristics of the opaque and glazed parts of the façade meet the minimum requirements set in the local national standards and are summarized in Table 1. The opaque portion of the façade is a typical curtain wall construction, while a concrete slab is adopted for the horizontal partitions (Table 1). The transparent portion of the South-oriented wall integrates an active smart glazing with the properties summarised in Table 2.

The test office room is flanked by identical offices on two sides on the same floor and on the floor immediately above and below, while the third side on the same floor is adjacent to a corridor space, with identical characteristics to the office rooms. Indoor comfort is considered as a requirement of the indoor space which is always met by the building services: indoor temperature has fixed setpoints for heating and cooling $\left(20{ }^{\circ} \mathrm{C}\right.$ and $26{ }^{\circ} \mathrm{C}$ respectively) with a nocturnal set-back $\left(12{ }^{\circ} \mathrm{C}\right.$ and $40{ }^{\circ} \mathrm{C}$ respectively); primary air ventilation rate is set to 1.4
$1 / \mathrm{sm} 2$ when the office is occupied; threshold of 320 lux is considered for the minimum illumination level, to be maintained by a combination of daylight and dimmable artificial lighting system (at desk level, $0.8 \mathrm{~m}$ high, at 1.5, R1, and 3.5 m, R2, far from the façade, cf. Figure 2).

Table 1: Characteristics of the opaque and glazed parts of the façade.

\begin{tabular}{|c|c|c|c|}
\hline Construction & Unit & $\begin{array}{c}\text { Curtain } \\
\text { wall }\end{array}$ & $\begin{array}{c}\text { Concrete } \\
\text { slab }\end{array}$ \\
\hline U-value glazing & {$\left[\mathrm{W} / \mathrm{m}^{2} \mathrm{~K}\right]$} & 2.00 & - \\
\hline U-value wall & {$\left[\mathrm{W} / \mathrm{m}^{2} \mathrm{~K}\right]$} & 0.27 & - \\
\hline $\begin{array}{c}\text { Internal thermal } \\
\text { capacity }\end{array}$ & {$\left[\mathrm{kJ} / \mathrm{m}^{2} \mathrm{~K}\right]$} & 21.7 & 67.8 \\
\hline $\begin{array}{c}\text { External thermal } \\
\text { capacity }\end{array}$ & {$\left[\mathrm{kJ} / \mathrm{m}^{2} \mathrm{~K}\right]$} & 23.2 & 29.3 \\
\hline Superficial mass & {$\left[\mathrm{kg} / \mathrm{m}^{2}\right]$} & 54 & 675 \\
\hline Time lag & {$[\mathrm{hrs}]$} & 1.63 & 10.61 \\
\hline
\end{tabular}

Table 2: Smart glazing properties.

\begin{tabular}{|c|c|c|}
\hline State & $\boldsymbol{\tau}_{\text {VIS }}[-]$ & g-value [-] \\
\hline 4 & 0.595 & 0.508 \\
\hline 3 & 0.446 & 0.396 \\
\hline 2 & 0.341 & 0.325 \\
\hline 1 & 0.238 & 0.238 \\
\hline
\end{tabular}

Schedules and peak loads for the building services, lighting, equipment and occupation are defined according to the ASHRAE standard 90.1 (ANSI/ASHRAE/IES, 2013). The lighting power density is set to $12.75 \mathrm{~W} / \mathrm{m} 2$, the equipment power density is $13.45 \mathrm{~W} / \mathrm{m} 2$, while the room is occupied by 2 people. A reversible heat pump is considered to provide heating and cooling to the office building, with an average seasonal COP of 3.5 for the winter and Seasonal Energy Efficiency Ratio of 2.5 for the summer. All energy uses are electrical therefore no conversion to primary energy is done. The smart glazing is controlled with three alternative reference controls, the first one is a reference rule-based control, while the last two represent a performance bound to the control devised with the present methodology, that is the maximum performance ideally achievable by controlling a smart glazing with the described properties. The smart glazing is controlled with the three alternative strategies below:

1) RBC-Passive: this is based on the amount of incident solar radiation on the façade as follows (Table 3):

Table 3: RBC reference control.

\begin{tabular}{|c|c|c|c|}
\hline State 4 & State 3 & State 2 & State 1 \\
\hline $0-100$ & $100-250$ & $250-700$ & $>=700$ \\
{$\left[\mathrm{~W} / \mathrm{m}^{2}\right]$} & {$\left[\mathrm{W} / \mathrm{m}^{2}\right]$} & $\mathrm{W} / \mathrm{m} 2$ & $\mathrm{~W} / \mathrm{m} 2$ \\
\hline
\end{tabular}

2) RBC - Opt Hourly: the smart glazing adopts a state, at 1-hour intervals, which minimizes the total building loads (sum of heating, cooling and lighting loads). 
This control option is the best performance achievable by means of an RBC controller aiming at minimizing energy use based on current and past boundary conditions. In order to implement this controller an optimisation problem needs to be solved for each hour of the simulation. The objective function adopted (2) for this optimisation is as follows:

$$
\min \left\{\begin{array}{c}
f(X)=\dot{Q}=\dot{Q}_{\text {heat }}+\dot{Q}_{\text {cool }}+\dot{Q}_{\text {ligh }}\left[\frac{k W}{m^{2} y}\right] \\
X(t)=\left(g-\text { value }(t)[-], \tau_{\text {vis }}(t)[-]\right)
\end{array}\right.
$$

3) MPC: the smart glazing is actively controlled at 1hour intervals, such that its control sequence minimizes the total site energy use of the building over a certain time horizon. The implementation of this controller relies on the solution of the following optimisation problem (3):

$\min \left\{\begin{array}{l}f(X)=S E=S E_{h}+S E_{c}+S E_{l}\left[\frac{k W h}{m^{2} y}\right] \\ X(t)=\left(g-\operatorname{value}(t)[-], \tau_{\text {vis }}(t)[-]\right)\end{array}\right.$

In order to optimise the RBC and MPC controls the simulation framework developed by Favoino et al. (2017) is adopted, so called AFast (Adaptive Façade advanced simulation tool). This simulation framework, specifically designed for multi-physical simulation of adaptive building envelope systems, connects multiple building models (i.e. thermal, daylight etc.) by means of a data integration strategy (Trcka et al, 2009), and optimises control strategy of building systems and envelope systems by adopting an optimisation engine. Within this tool the overall simulation, which is constituted by different subsimulations (relative to different models and consequential days), is managed by means of a MATLAB middle-ware. In particular, for RBC control the planning and cost horizon coincide (i.e. 1 hour), while for the MPC control a planning horizon of 1 day and a cost horizon of 3 days are considered, in order to account for building dynamics (time constant of the building system, weekend occupation and variable set-points), as suggested by Corbin et al. (2013). The length of the pre-conditioning horizon was set to two months in order to minimise the effect of the adaptive façade control on the optimisation results. A hybrid optimisation algorithm (GPSPSOCCHJ) is adopted for the optimisation (Wetter, 2011), as it offers the best trade-off between computational time and optimality of the results when compared with alternative algorithms. For details of the optimisation parameters please refer (Favoino et al. 2016).

\section{Results}

The methodological framework presented in section 2 was applied on the previously mentioned case study. The results of the rule extraction phase (i.e. decision tree 1 and decision tree 2) are presented in this section in order to assess the performance of both decision trees achieved in the open and closed loop test.

In the open loop test the classification accuracy $\mathrm{A}_{\mathrm{dt}}$ of the decision trees, has been employed as performance measure suitable for classification and categorical forecasting. The classification accuracy $A_{d t}$ is calculated as follow (4):

$$
A_{d t}=\frac{n_{\text {correct }}^{\circ}}{n^{\circ}{ }_{\text {tot }}}
$$

Where $\mathrm{n}^{\circ}$ correct is the number of hourly discrete states of the smart glazing correctly predicted and $\mathrm{n}^{\circ}$ tot is the number of statistical observations in one year without considering the night hours (i.e., $8760-3285=5475$ ). The two models were developed using different pools of input variables, but for the sake of comparability the same splitting, stopping and pruning criteria have been considered.

In particular, the tree splitting procedure is based on the reduction of the GINI impurity measure (Tan et al. 2005) until the stopping criterion has been satisfied. In this specific case study, the selected stopping criterion was based on the minimum number of observations in the child nodes of the tree (i.e., 10 observations).

\begin{tabular}{|c|c|c|c|c|c|c|}
\hline D.tree & Variable & Description & $\begin{array}{c}\text { Backward lag } \\
{[\mathrm{hrs}]}\end{array}$ & $\begin{array}{c}\text { Forward lag } \\
{[\mathrm{hrs}]}\end{array}$ & $\mathbf{A}_{d t}$ & $\begin{array}{c}\mathbf{n}^{\circ} \\
\text { rules }\end{array}$ \\
\hline \multirow{5}{*}{1} & $\mathrm{~T}_{\mathrm{int}, \mathrm{h}}$ & Hourly Indoor zone temperature $\left[{ }^{\circ} \mathrm{C}\right]$ & -1 & - & \multirow{5}{*}{$61 \%$} & \multirow{5}{*}{21} \\
\hline & $\mathrm{T}_{\text {est } \mathrm{h}}$ & Hourly outdoor temperature $\left[{ }^{\circ} \mathrm{C}\right]$ & $-21,-37,-42$ & - & & \\
\hline & $\mathrm{SR}_{\mathrm{h}}$ & Hourly solar radiation $\left[\mathrm{W} / \mathrm{m}^{2}\right]$ & $\begin{array}{c}-1,-8,-13,-23,-24,- \\
33,-36,-37,-42 \\
\end{array}$ & - & & \\
\hline & $T_{\text {est,day }}$ & Daily average outdoor temperature $\left[{ }^{\circ} \mathrm{C}\right]$ & - & - & & \\
\hline & hour & Hour of the day & - & - & & \\
\hline \multirow{5}{*}{2} & $\mathrm{~T}_{\mathrm{int}, \mathrm{h}}$ & Hourly Indoor zone temperature $\left[{ }^{\circ} \mathrm{C}\right]$ & -1 & - & \multirow{5}{*}{$64 \%$} & \multirow{5}{*}{28} \\
\hline & $\mathrm{T}_{\text {est,h }}$ & Hourly outdoor temperature $\left[{ }^{\circ} \mathrm{C}\right]$ & $-3,-42$ & +3 & & \\
\hline & $\mathrm{SR}_{\mathrm{h}}$ & Hourly solar radiation $\left[\mathrm{W} / \mathrm{m}^{2}\right]$ & $\begin{array}{l}-1,-10,-12,-13 \\
-17,-24,-37,-41 \\
\end{array}$ & $\begin{array}{c}0,+1,+2,+3 \\
+4\end{array}$ & & \\
\hline & Test,day & Daily average outdoor temperature $\left[{ }^{\circ} \mathrm{C}\right]$ & - & - & & \\
\hline & hour & Hour of the day & - & - & & \\
\hline
\end{tabular}

Table 4: input variables and performances of the two classifiers. 
In addition, the cost-complexity pruning process (i.e., 1SE rule) was performed for both models (Capozzoli et al. 2018). This procedure makes it possible to identify the optimal value of the tuning parameter $\alpha$ in order to prune the original tree obtained by a k-fold cross validation as explained in section 3 .

Table 4 summarises the input variables considered in the model development, the number of control rules extracted, and the overall performance of the classifiers achieved in the open loop test. Both trees were trained and tested using the same variables but with different lags (automatically selected from the algorithm).

In particular, for the decision tree 1 , the algorithm was free to select as predictive attributes, variables with a maximum backward lag of 48 hours (i.e., $\mathrm{T}_{\mathrm{int}, \mathrm{h}}, \mathrm{T}_{\mathrm{est}, \mathrm{h}}$, $\left.\mathrm{SR}_{\mathrm{h}}\right)$.

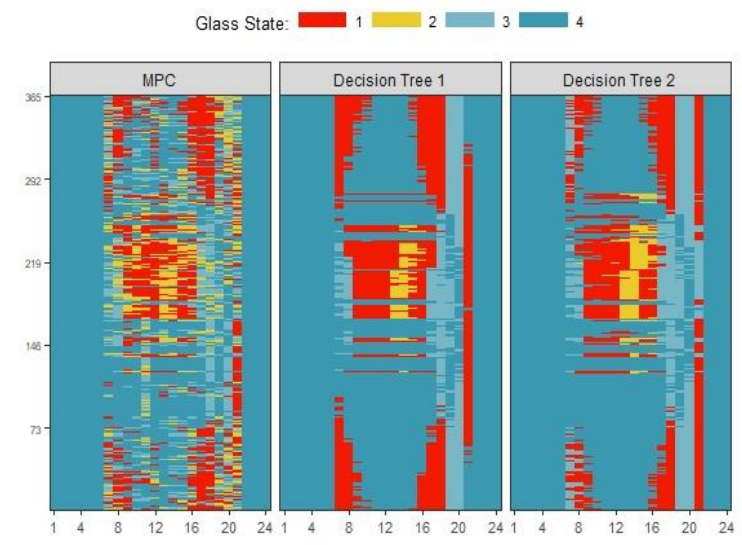

Figure 3: Carpet plots of the hourly control signal of

the MPC (a) decision tree $1(b)$ and decision tree 2 (c)

However, the best performance was achieved by using only few shifted signals. In the same way, the decision tree 2, was developed assuming, for the exogenous variables (i.e., $\mathrm{T}_{\text {est, }}, \mathrm{SR}_{\mathrm{h}}$ ), also a maximum forward lag of 6 hours (compared to the $48 \mathrm{~h}$ perfect forecast of the MPC). The amplitude of the maximum forward time lag was intentionally limited in order to make its use more reasonable in a real-life implementation. In the open loop test the classifiers perform similarly, with an accuracy of the decision tree 2 slightly higher. This is due to the fact that the forward-lagged tree is capable to better learn the control logic (with a higher number of decision rules) of the MPC that, by its nature, leverages on disturbance prediction for providing the optimal control policy.

Figure 3 shows the carpet plots of the hourly control signal of the MPC, decision tree 1 and decision tree 2 . The classifiers are able to reproduce the most significant logics of the MPC controller while simultaneously reducing the number of state changes of the smart glazing during the day. The final stage of the analysis is aimed at comparing the extracted control logics versus the MPC and other rule-based controllers through a closed loop test, by embedding them in the building energy model.

To this sake the extracted rules are implemented in the EnergyPlus model by means of the Energy Management System tool (EMS) (DOE U.S. 2015). Particularly past states were coded as "Trend Variables", while predictions are interrogated as schedules of boundary conditions with perfect knowledge (no uncertainties in the prediction, as per the simulated MPC benchmark). The implementation of the extracted rules through the EMS represents the closest approximation of the controller performance on a real automation system.

In fact, the misclassification error made by the controller could cause the occurrence of indoor conditions different from those experienced during the training phase generating a divergence from the control sequence reproduced in the open loop test (Figure 3). Thanks to this analysis it is possible to assess the impact of the misclassification error on the global energy performance.

The performance benchmark of the rule extracted with the presented methodology is the energy use resulting by operating the smart glazing with i) $\mathrm{RBC}$ - passive control control; ii) RBC - Opt Hourly control; iii) MPC control; previously introduced in Section Model and Case study.

The results obtained (Table 5) show that the relatively poor learning performances achieved by the two classifiers in the open loop test, correspond to an energy performance (in terms of global primary energy) very close to the one of the MPC controller. In addition, both decision trees perform better than the reference RBC controllers (i.e., passive and $\mathrm{H}$ opt), suggesting that the rule extraction process from MPC (even using the only backward-lagged variables - decision tree 1) can successfully lead to an improvement of the standard rulebased control logics for smart glazing technologies. The most promising result is achieved by decision tree 2 ,

Table 5: Performance comparison between RBCs, MPC and MPC-enhanced RBC controls of smart glazing.

\begin{tabular}{|l|c|c|c|c|c|c|c|c|c|}
\hline \multirow{2}{*}{$\begin{array}{c}\text { Control } \\
\text { type }\end{array}$} & \multicolumn{4}{|c|}{ Site Energy Uses $\left[\mathrm{kWh} / \mathrm{m}^{2} \mathrm{y}\right]$} & \multicolumn{4}{c|}{ Performance Reduction vs MPC } \\
\cline { 2 - 10 } & SE heat & SE cool & SE light & SE gl & $\begin{array}{c}\mathbf{N}^{\circ} \text { state } \\
\text { change } \\
{[-]}\end{array}$ & $\begin{array}{c}\text { SE heat } \\
{[\%]}\end{array}$ & $\begin{array}{c}\text { SE cool } \\
{[\%]}\end{array}$ & $\begin{array}{c}\text { SE light } \\
{[\%]}\end{array}$ & $\begin{array}{c}\text { SE gl } \\
{[\%]}\end{array}$ \\
\hline Passive & 20.66 & 1.47 & 7.59 & 29.73 & 1428 & - & - & - & - \\
\hline H opt & 19.17 & 1.22 & 8.14 & 28.52 & 743 & - & - & - & - \\
\hline MPC & 18.96 & 1.09 & 5.29 & 25.33 & 3153 & - & - & - & - \\
\hline $\begin{array}{l}\text { Decision } \\
\text { tree 1 }\end{array}$ & 18.16 & 1.41 & 7.41 & 26.98 & 2039 & $4.19 \%$ & $-29.70 \%$ & $-40.12 \%$ & $-6.51 \%$ \\
\hline $\begin{array}{l}\text { Decision } \\
\text { tree 2 }\end{array}$ & 18.90 & 1.19 & 5.48 & 25.57 & 2293 & $0.30 \%$ & $-9.33 \%$ & $-3.71 \%$ & $-0.95 \%$ \\
\hline
\end{tabular}


whose global primary energy demand (expressed in $\mathrm{kWh} / \mathrm{m}^{2} \mathrm{y}$ ) deviates from the MPC one of the $-0.95 \%$ and from the best RBC reference (i.e., $\mathrm{H}$ opt control) of the $+11.54 \%$.

Figure 4 shows the daily percentage differences between MPC and decision tree 2, for each energy use during the simulated reference year. The solid lines represent the ratio between the daily and yearly primary energy demand for heating, cooling and lighting achieved of the MPC controller. The dashed lines instead, represent the daily percentage difference between MPC and decision tree 2 for each energy use normalised on the total MPC energy use. It is possible to see that differences occur mainly regarding heating and lighting energy uses, despite some peak differences in cooling. During the middle season the MPC seems to perform better than the decision tree due to MPC is capable to better balance the room energy demand through an effective modulation of the entering solar radiation, minimizing the number of hours during which the HVAC and lighting systems are operated. While, as discussed in Favoino et al. (2016), during more extreme climate conditions, simple rules may be effective as the MPC control.

\section{Discussion and conclusions}

This paper has focused on the advanced control of a smart glazing technology. The main objective has been to investigate the effectiveness of a data-driven rule extraction procedure for reproducing the optimal control policy provided by an ideal MPC. To the best of authors knowledge this is the first attempt for the approximation of MPC logics for electrochromic switchable glazing or more in general to modulate free solar gains through building envelope. Differently from similar applications tested for other systems, the approximation of the MPC via decision tree is formulated as a multinomial classification problem since that the considered smart glazing can adopt four discrete states.

In addition, the control logics of the advanced RBC controllers have been extracted from a reference control period of one year. This last assumption increased the complexity of the classification task since the model should extract a set of "if-then" control rules for the glazing, by experiencing indoor and outdoor conditions that could significantly change among the year. On the other hand, through this approach it was possible to develop one yearly model instead of seasonal ones for each characteristic climate condition. In this way there is no uncertainty in selecting the set of control rules to be used during operation.

In order to assess the potentials of the developed RBC controllers, both open and closed loop tests have been performed. The performances achieved in the open loop test are in the range of $60-65 \%$ of model accuracy in reproducing the MPC control signal. Comparing the obtained results with the literature the classifiers perform poorly. By the way the performances achieved in the closed loop tests are promising given that the percentage difference between the MPC and decision tree 1 and 2 in terms of global primary energy demand are of $-6.51 \%$ and $-0.95 \%$ respectively. There are two main reasons behind this: i) the MPC puts too much effort in switching the glazing even though the impact of the control action is not so relevant, ii) the previous control states of the glazing are not considered as input variables of the classifiers. Specifically, the selection of the previous control signals as predictive attributes could have improved the performance in the open loop test but at the same time can generate a higher divergence from the optimal solution in the closed loop test. In fact, the misclassification error made by an autoregressive controller could cause the occurrence of indoor conditions different from those learned during the training phase affecting the control action both in the current and future timesteps.

Summarizing, the present study demonstrated that the rule extraction approach can successfully reproduce sophisticated control logics ensuring complexity reduction without losing key information. In this perspective future works will be aimed at simulating the MPC and RBC controllers in a more realistic way (i.e., without perfect prediction of the disturbances) and implementing them on real experimental facilities. In addition, the generalizability (to other case studies) and

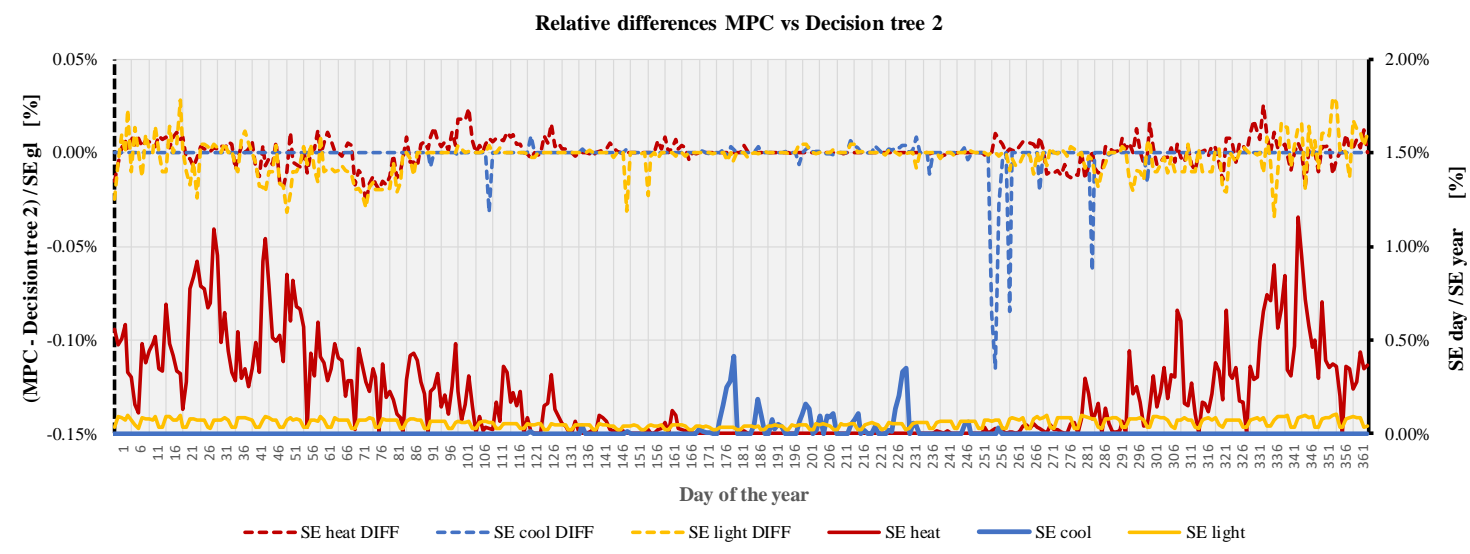

Figure 4: Daily percentage differences between MPC and decision tree 2 for each energy use. 
scalability (from single window to an entire façade) of advanced RBC controllers will be subjects of further investigations.

\section{References}

Aelenei, D., Aelenei, L., Loonen, R., Perino, M., Serra, V. (edit by Asdrubali, F., Desideri, U.). (2019). Chapter 6 - Building Envelope in Handbook of Energy Efficiency in Buildings: A Life Cycle Approach. Butterworth-Heinemann, Oxford (UK).

Assimakopoulos, M.N., Tsangrassoulis, A., Guarracino G., Santamouris, M., (2004). Integrated energetic approach for a controllable electrochromic device. Energy and Buildings 36(5), 415-422.

Assimakopoulos, M.N., Tsangrassoulis, A., Santamouris, M., Guarracino, G. (2007). Comparing the energy performance of an electrochromic window under various control strategies. Building and Environment, 42, 2829-2834.

Capozzoli, A., Piscitelli, M.S., Brandi, S., Grassi, D., Chicco, G. (2018). Automated load pattern learning and anomaly detection for enhancing energy management in smart buildings. Energy 157, 336-352.

Capozzoli, A., Piscitelli, M.S., Neri, F., Grassi, D., Serale, G. (2016). A novel methodology for energy performance benchmarking of buildings by means of Linear Mixed Effect Model: the case of space and DHW heating of out-patient Healthcare Centres. Applied Energy 171, 592-607.

Capozzoli, A., Grassi, D., Causone, F. (2015). Estimation models of heating energy consumption in schools for local authorities planning. Energy and Buildings 105, 302-313.

Corbin, C.D., Henze, G.P., May-Ostendorp, P. (2013). A model predictive control optimization environment for real-time commercial building application. Journal of Building Performance Simulation 6(3), 159-174.

DOE U.S. (2015). Application Guide for EMS Energy Management System - User Guide.

DOE U.S. (2014). EnergyPlus Engineering Reference, Office of Energy Efficiency and Renewable Energy, United States

Dussault, J.M., Gosselin, L., Galstian, T. (2012). Integration of smart windows into building design for reduction of yearly overall energy consumption and peak loads. Solar Energy 86(11), 3405-3416.

Favoino, F., Fiorito, F., Cannavale, A., Ranzi, G., Overend, M. (2016). Optimal control and performance of photovoltachromic switchable glazing for building integration in temperate climates. Applied Energy 178, 943-961.

Favoino, F. (2017). Building Performance Simulation of Adaptive Facades, $\mathrm{PhD}$ thesis, University of Cambridge.
Gugliermetti, F., Bisegna, F. (2003). Visual and energy management of electrochromic windows in Mediterranean climate. Building and Environment 38, 479-492.

Granqvist, C.G. (2016). Electrochromics and Thermochromics: Towards a New Paradigm for Energy Efficient Buildings. Materials Today: Proceedings (3)1, 2-11.

Jin, Q., Favoino, F., Overend, M. (2017). Design and control optimisation of adaptive insulation systems for office buildings. Part 2: A parametric study for a temperate climate. Energy 127, 634-649.

Jonsson, A., Roos, A. (2010). Evaluation of control strategies for different smart window combinations using computer simulations. Solar Energy 84(1), 1-9.

Lee, E.S., Claybaugh, E.S., LaFrance, M. (2012). End user impacts of automated electrochromic windows in a pilot retrofit application. Energy and Buildings 47, 267-284,

Lee, E. S., Tavil, A. (2007). Energy and visual comfort performance of electrochromic windows with overhangs. Building and Environment 42(6), 24392449.

Ma, Y., Matusko, J., Borrelli, F. (2015). Stochastic model predictive control for building HVAC systems: Complexity and conservatism. IEEE Transactions on Control Systems Technology 23(1), 101-116

May-Ostendorp, P., Henze, G.P., Corbin, C.D., Rajagopalan, B., Felsmann, C. (2011). Modelpredictive control of mixed-mode buildings with rule extraction. Building and Environment 46(2), 428-437.

Oldewurtel, F., Parisio, A., Jones, C.N., Gyalistras, D., Gwerder, M., Stauch, V., Lehmann, B., Morari, M., (2012). Use of model predictive control and weather forecasts for energy efficient building climate control. Energy and Buildings 45, 15-27

Serale, G., Fiorentini, M., Capozzoli, A., Bernardini, D., Bemporad, A. (2018). Model Predictive Control (MPC) for Enhancing Building and HVAC System Energy Efficiency: Problem Formulation, Applications and Opportunities. Energies 11(3), 631.

Tan, P., Steinbach, M., Kumar, V. (2005). Introduction to data mining. Pearson Addison-Wesley. Boston (MA).

Trcka, M., Hensen, J.L.M., Wetter, M. (2009). "CoSimulation of Innovative Integrated HVAC Systems in Buildings. Journal of Building Performance Simulation 2 (3), 209-30.

Yan, R., Ma, Z., Zhao, Y. (2016). Kokogiannakis G. A decision tree based data-driven diagnostic strategy for air handling units. Energy and Buildings 133, 37-45.

Wetter, M. (edit by Simulation Research Group, Lawrence Berkeley National Laboratory). (2011). GenOpt Generic Optimization program User Manual, Version 3.1.0. Berkeley (CA) 